\title{
FIXED POINT ITERATION FOR LOCAL STRICTLY PSEUDO-CONTRACTIVE MAPPING
}

\author{
XINLONG WENG
}

(Communicated by Paul S. Muhly)

\begin{abstract}
A fixed point of the local strictly pseudo-contractive mapping is obtained as the limit of an iteratively constructed sequence with an error estimation in uniformly smooth Banach spaces.
\end{abstract}

This paper has been motivated by a paper by C. E. Chidume [2], in which an iterative approximation of the fixed point of a Lipschitzian strictly pseudocontractive mapping in $L_{p}(2 \leq p<\infty)$ space is developed. The object of the present note is to consider the local version of the problem and to extend Chidume's results to general uniformly smooth Banach spaces. Furthermore, we replace strictly pseudo-contractive mapping by local strictly pseudo-contractive mapping and the Lipschitzian condition by a weaker assumption.

Let $X$ be a Banach space, a mapping $T$ is said to be local strictly pseudocontractive if for any $x \in D(T)$ there exists a number $t_{x}>1$ such that the inequality

$$
\|x-y\| \leq\left\|(1+r)(x-y)-r t_{x}(T x-T y)\right\|
$$

holds for all $y \in D(T)$ and $r>0$. We denote by $J$ the normalized duality mapping from $X$ to $2^{X^{*}}$ given by

$$
J x=\left\{f^{*} \in X^{*}:\left\|f^{*}\right\|^{2}=\|x\|^{2}=\operatorname{Re}\left\langle x, f^{*}\right\rangle\right\}
$$

where $\langle\cdot, \cdot\rangle$ denotes the generalized duality pairing. If $X$ is uniformly smooth, then $J$ is single-valued and uniformly continuous on bounded set. A mapping $T$ is said to be local strongly accretive if given $x \in D(T)$ there exists a positive number $k_{x}$ such that for each $y \in D(T)$ there is $j \in J(x-y)$ such that

$$
\langle T x-T y, j\rangle \geq k_{x}\|x-y\|^{2} .
$$

In [3] T. Kato discovered the relationship between pseudo-contractive mappings

Received by the editors April 23, 1990.

1980 Mathematics Subject Classification (1985 Revision). Primary 47H15; Secondary 47H05.

Key words and phrases. Local strictly pseudo-contractive mapping, iterative method. 
and accretive mappings, proving

Lemma 1 [3]. Let $x, y \in X$. Then $\|x\| \leq\|x+\alpha y\|$ for every $\alpha>0$ if and only if there is $f \in J x$ such that $\operatorname{Re}\langle y, f\rangle \geq 0$.

Applying Lemma 1, we get

Lemma 2. Let $X$ be a Banach space, $K$ a subset of $X$, and $U: K \mapsto X$. Then $U$ is a local strictly pseudo-contractive mapping if and only if $T=I-U$ is a local strongly accretive mapping.

Proof. If $U$ is a local strictly pseudo-contractive mapping, then given any $x \in$ $K$ there exists a number $t_{x}>1$ such that for any $y \in K, r>0$ we have

$$
\begin{aligned}
\|x-y\| & \leq\left\|(1+r)(x-y)-r t_{x}(U x-U y)\right\| \\
& =\left\|(1+r)(x-y)-r\left(t_{x} U x-t_{x} U y\right)\right\| \\
& =\left\|x-y+r\left(x-t_{x} U x-y+t_{x} U y\right)\right\| .
\end{aligned}
$$

By Lemma 1, there exists $j \in J(x-y)$ such that

$$
\operatorname{Re}\left\langle x-t_{x} U x-y+t_{x} U y, j\right\rangle \geq 0 .
$$

Observe that operator $I-t_{x} U=I-t_{x}(I-T)=t_{x} T-\left(t_{x}-1\right) I$ we obtain

$$
t_{x} \operatorname{Re}\langle T x-T y, j\rangle-\left(t_{x}-1\right) \operatorname{Re}\langle x-y, j\rangle \geq 0,
$$

which produces

$$
\operatorname{Re}\langle T x-T y, j\rangle \geq\left(1-1 / t_{x}\right)\|x-y\|^{2}
$$

for all $y \in K$, i.e., $T$ is a local strongly accretive mapping. Suppose, conversely, that $T$ is local strongly accretive. Then for any given $x \in K$ there exists a number $k_{x}>0$ and for $y \in K$ there exists $j \in J(x-y)$ such that

$$
\operatorname{Re}\langle T x-T y, j\rangle \geq k_{x}\|x-y\|^{2} .
$$

Without loss of generality, we can assume $1>k_{x}>0$, and let $t_{x}=1 /\left(1-k_{x}\right)$. Then the above inequality becomes

$$
\operatorname{Re}\langle T x-T y, j\rangle \geq\left(1-1 / t_{x}\right)\|x-y\|^{2} .
$$

By the same proof as above but in the reverse order, we get that $U$ is a local strictly pseudo-contractive mapping.

Let $X$ be an arbitrary Banach space with $\operatorname{dim} X \geq 2$, the modulus of convexity $\delta_{X}(\varepsilon), 0<\varepsilon \leq 2$, of $X$ is defined by

$$
\delta_{X}(\varepsilon)=\inf \{1-\|x+y\| / 2: x, y \in X,\|x\|=\|y\|=1,\|x-y\|=\varepsilon\} .
$$

$X$ is said to be uniformly convex if $\delta_{X}(\varepsilon)>0$ for every $\varepsilon>0$ and uniformly smooth if the dual space $X^{*}$ is uniformly convex. The estimation of the modulus of convexity for the spaces $L^{p}, e^{p}, W_{m}^{p}, 1<p<\infty$ are [1]:

$$
\begin{array}{cc}
\delta_{X}(\varepsilon) \geq(p-1 / 16) \varepsilon^{2}, & 1<p \leq 2, \\
\delta_{X}(\varepsilon) \geq p^{-1}(\varepsilon / 2)^{p}, & p \geq 2 .
\end{array}
$$


We define for positive $t$

$$
\beta(t)=\sup \left\{\left(\|x+t y\|^{2}-\|x\|^{2}\right) / t-2 \operatorname{Re}\langle y, J(x)\rangle:\|x\| \leq 1,\|y\| \leq 1\right\}
$$

Clearly $\beta:(0, \infty) \rightarrow[0, \infty)$ is nondecreasing, continuous and $\beta(c t) \leq c \beta(t)$ for $c \geq 1$. Also we have

Lemma 3 [4]. If $X$ is a uniformly smooth Banach space and $\beta(t)$ is defined as above, then $\lim _{t \rightarrow 0+} \beta(t)=0$ and

$$
\|x+y\|^{2} \leq\|x\|^{2}+2 \operatorname{Re}\langle y, J(x)\rangle+\max \{\|x\|, 1\}\|y\| \beta(\|y\|)
$$

for all $x, y \in X$.

We also need the following:

Lemma 4. Let $\beta_{n}$ be a nonnegative sequence satisfying

$$
\beta_{n+1} \leq\left(1-\delta_{n}\right) \beta_{n}+\sigma_{n}
$$

with $\delta_{n} \in[0,1], \sum_{i=1}^{\infty} \delta_{i}=\infty$, and $\sigma_{n}=o\left(\delta_{n}\right)$. Then $\lim _{n \rightarrow \infty} \beta_{n}=0$.

Proof. Since $\sigma_{n}=o\left(\delta_{n}\right)$, let $\sigma_{n}=\varepsilon_{n} \cdot \delta_{n}$, and $\varepsilon_{n} \rightarrow 0$. By a straightforward induction, one obtains

$$
0 \leq \beta_{n+1} \leq \prod_{j=k}^{n}\left(1-\delta_{j}\right) \beta_{k}+\sum_{j=k}^{n}\left[\delta_{j} \prod_{i=j+1}^{n}\left(1-\delta_{i}\right)\right] \varepsilon_{j}
$$

We have

$$
\prod_{j=k}^{n}\left(1-\delta_{j}\right) \leq e^{-\sum_{j=k}^{n} \delta_{j}} \rightarrow 0
$$

and

$$
\sum_{j=k}^{n} \delta_{j} \prod_{i=j+1}^{n}\left(1-\delta_{i}\right) \leq 1 \quad \forall n, k
$$

Given $\varepsilon>0$, pick $k$ such that $\varepsilon_{j} \leq \varepsilon$ for all $j \geq k$, from $(*)$ we have

$$
0 \leq \liminf \beta_{n} \leq \lim \sup \beta_{n} \leq \varepsilon \text {. }
$$

Letting $\varepsilon \rightarrow 0$, we obtain $\lim _{n \rightarrow \infty} \beta_{n}=0$.

For the rest of this note, $F(U)$ denotes the set of fixed points of the mapping $U$.

Theorem 1. Let $K$ be a subset of a uniformly smooth Banach space and $U: K \mapsto$ $X$ be a local strictly pseudo-contractive mapping. If $F(U) \neq \varnothing$ and the range of $U$ is bounded, then $\left\{x_{n}\right\} \subset K$ generated by $x_{1} \in K$,

$$
x_{n+1}=\left(1-C_{n}\right) x_{n}+C_{n} U x_{n}
$$

with $\left\{C_{n}\right\} \subset(0,1]$, satisfying

$$
\sum_{n=1}^{\infty} C_{n}=\infty, \quad C_{n} \rightarrow 0
$$

strongly converges to $p \in F(U)$ and $F(U)$ is a single set. 
Proof. Let $p$ be a fixed point of $U$. Since $U$ is local strictly pseudo contractive mapping, then $T=I-U$ is local strongly accretive. Thus, there exists a positive number $k_{p}$ such that for each $x_{n} \in K$

$$
\operatorname{Re}\left\langle x_{n}-U x_{n}-p+U p, J\left(x_{n}-p\right)\right\rangle \geq k_{p}\left\|x_{n}-p\right\|^{2} .
$$

Now set

$$
\beta_{n}=\left\|x_{n}-p\right\|^{2}
$$

and

$$
d=\sup \{\|U x-p\|: x \in K\} .
$$

Because $C_{n} \rightarrow 0$, it is easy to show there exists an integer $N \geq 1$ such that when $n \geq N$

$$
\left[1-k_{p} C_{n}\right]^{2}+d^{2} C_{n} \beta\left(C_{n}\right) \leq 1 .
$$

Let $B=\max \left\{\beta_{i}: 1 \leq i \leq N, 1\right\}$. First we want to show $\beta_{n} \leq B^{2}$, and

$$
\beta_{n+1} \leq\left[1-k_{p} C_{n}\right]^{2} \beta_{n}+B^{2} d^{2} C_{n} \beta\left(C_{n}\right) \text {. }
$$

From the definition of $\left\{x_{n}\right\}$ and Lemma 3, we have

$$
\begin{aligned}
\beta_{n+1}= & \left\|x_{n+1}-p\right\|^{2}=\left\|\left(1-C_{n}\right)\left(x_{n}-p\right)+C_{n}\left(U x_{n}-p\right)\right\|^{2} \\
\leq & \left(1-C_{n}\right)^{2}\left\|x_{n}-p\right\|^{2}+2 C_{n}\left(1-C_{n}\right) \operatorname{Re}\left\langle U x_{n}-p, J\left(x_{n}-p\right)\right\rangle \\
& +\max \left\{\left\|x_{n}-p\right\|, 1\right\} C_{n}\left\|U x_{n}-p\right\| \beta\left(C_{n}\left\|U x_{n}-p\right\|\right) \\
\leq & \left(1-C_{n}\right)^{2} \beta_{n}+\max \left\{\left\|x_{n}-p\right\|, 1\right\} d^{2} C_{n} \beta\left(C_{n}\right) \\
& +2 C_{n}\left(1-C_{n}\right)\left\|x_{n}-p\right\|^{2}-2 C_{n}\left(1-C_{n}\right) \\
& \times \operatorname{Re}\left\langle x_{n}-U x_{n}-p+U p, J\left(x_{n}-p\right)\right\rangle \\
\leq & {\left[\left(1-C_{n}\right)^{2}+2\left(1-k_{p}\right) C_{n}\left(1-C_{n}\right)\right] \beta_{n}+\max \left\{\left\|x_{n}-p\right\|, 1\right\} d^{2} C_{n} \beta\left(C_{n}\right) } \\
\leq & {\left[1-k_{p} C_{n}\right]^{2} \beta_{n}+\max \left\{\beta_{n}, 1\right\} d^{2} C_{n} \beta\left(C_{n}\right) . }
\end{aligned}
$$

If $n \leq N$, according the definition of number $B$, so $\beta_{n} \leq B^{2}$. For $n \geq N$ apply induction: assume $\beta_{n} \leq B^{2}$ then

$$
\begin{aligned}
\beta_{n+1} & \leq\left[1-k_{p} C_{n}\right]^{2} \beta_{n}+B^{2} d^{2} C_{n} \beta\left(C_{n}\right) \\
& \leq\left\{\left[1-k_{p} C_{n}\right]^{2}+d^{2} C_{n} \beta\left(C_{n}\right)\right\} B^{2} \leq B^{2},
\end{aligned}
$$

so we obtain $\beta_{n} \leq B^{2}$ for all $n$ and from above inequality we get $(* *)$. Now applying Lemma 4 to inequality $(* *)$ we get that $\left\{x_{n}\right\}$ strongly converges to the fixed point $p$. Now suppose there is a $p^{*} \in F(U)$ with $p^{*} \neq p$. Repeating the argument of the theorem relative to $p^{*}$, one sees that $\left\{x_{n}\right\}$ converges to both $p^{*}$ and $p$, showing that $F(U)=\{p\}$.

For the special Banach spaces $L^{p}, e^{p}, W_{m}^{p}, 1<p<\infty$, we have the estimate [5]:

$$
\beta(t) \leq M t^{s-1},
$$


$s=2$ if $2 \leq p<\infty, s=p$ if $1<p<2$, and $M$ is some constant. Then we may able to obtain a convergence rate in the setting of Theorem 1 .

Theorem 2. Let $X=L^{p}, e^{p}, W_{m}^{p}, 1<p<\infty$, and $U,\left\{x_{n}\right\}, C_{n}$ be as in Theorem 1. Then we can find $\left\{C_{n}^{*}\right\}$ such that for sequence $\left\{x_{n}^{*}\right\}$, we have the estimate

$$
\left\|x_{n}^{*}-p\right\| \leq O\left(1 / n^{(s-1) / 2}\right),
$$

where $s=2$ if $2 \leq p<\infty, s=p$ if $1<p<2$.

Proof. Let

$$
\begin{aligned}
C_{n}^{*} & =\frac{k_{p}^{1 /(s-1)}}{1+n k_{p}^{s / s(s-1)},} \\
x_{n+1}^{*} & =\left(1-C_{n}^{*}\right) x_{n}^{*}+C_{n}^{*} U x_{n}^{*}, \\
\beta_{n}^{*} & =\left\|x_{n}^{*}-p\right\|^{2},
\end{aligned}
$$

and

$$
\alpha_{n+1}^{*}=\left[1-k_{p} C_{n}^{*}\right]^{s} \alpha_{n}^{*}+C_{n}^{* s}, \quad \alpha_{1}^{*}=\left(M B^{2} d^{2}\right)^{-1} \beta_{1}^{*} .
$$

From (**) and the estimation of $\beta(t)$ in $X$ spaces, we have $\beta_{n}^{*} \leq \alpha_{n}^{*}, n=$ $1,2, \ldots$. Solving the above equations we get

$$
\alpha_{n}^{*}=\alpha_{1}^{*} /\left[1+(n-1) \alpha_{1}^{* 1 /(s-1)}\left(k_{p}\right)^{s /(s-1)}\right]^{s-1} .
$$

The proof is complete.

\section{REFERENCES}

1. Ya. I. Al'ber and A. I. Notik, Geometric properties of Banach spaces and approximate methods for solving nonlinear operator equations, Soviet Math. Dokl. 29 (1984), 611-615.

2. C. E. Chidume, Iterative approximation of fixed points of Lipschitzian strictly pseudocontractive mappings, Proc. Amer. Math. Soc. 99 (1987), 283-288.

3. T. Kato, Nonlinear semigroups and evolution equations, J. Math. Soc. Japan 19 (1967), 509-519.

4. S. Reich, An iterative procedure for constructing zeros of accretive sets in Banach spaces, J. Nonlinear Anal. 2 (1978), 85-92.

5. __ Constructive techniques for accretive and monotone operators, Appl. Nonlinear Anal. Arlington, TX, 1979.

Department of Mathematics, Marshall University, Huntington, West Virginia 25755 